\title{
Integrative Mechanisms Controlling Directional Sensitivity of an Identified Sensory Interneuron
}

\author{
Gwen A. Jacobs, ${ }^{*}$ John P. Miller, ${ }^{*}$ and R. K. Murphey $\dagger$ \\ *Department of Zoology, University of California, Berkeley, California 94720, and †Department of Biology, SUNY, \\ Albany, Albany, New York 12222
}

Several identified interneurons in the cricket cercal afferent system display directional sensitivity to wind stimuli: the spike frequency of these cells depends on the wind direction with respect to the animal's body. Factors determining the directional sensitivity of one of these identified interneurons (interneuron 10-3) were studied in detail. This cell has 3 dendritic branches that arborize in 3 distinct regions of the terminal abdominal ganglion. Using 2 independent methods, it was demonstrated that the dendrites have different receptive fields to wind stimuli. First, small patches of filiform hairs, whose afferents projected to individual dendrites, were isolated and selectively stimulated. In each case the response of the cell matched the receptive field of the afferents in the patch. Second, a laser beam directed through the sterco dissecting microscope was used to photoinactivate small portions of the cell in situ during intracellular recording. By isolating or ablating individual dendrites, the contributions of each of the 3 dendrites to the overall receptive field were assessed. Although the receptive field of the whole cell could be predicted by a summation of the receptive fields of all 3 dendrites, the precise directional sensitivity of the cell could not be predicted by a simple linear summation of the receptive fields of each dendrite. Two factors were found to account for this nonlinearity of summation. The first factor was polysynaptic inhibition from other interneurons within the terminal abdominal ganglion. Wind directions that activate inhibition in interneuron 10-3 were identified, and the specific classes of filiform afferents that activate the inhibitory pathway were determined. The net effect of the inhibition was to "sharpen" the directional sensitivity of 10-3 by selectively decreasing the cell's response to specific excitatory inputs. The second factor that contributed to directional sensitivity was the complex electroanatomy of the interneuron. The probable location of the spikeinitiating zone (SIZ) was determined by using the laser photoinactivation technique. The relative efficacies of synaptic inputs onto the 3 different branches were then interpreted with respect to their different electrotonic distances from the SIZ. On the basis of the data obtained in this report, we present a qualitative model for the basis of directional sensitivity in this cell.

The relationship between the structural and functional properties of neurons has long interested theoretical and experimental neurobiologists. In very few instances, however, has it been possible to test directly how the distribution of excitatory and inhibitory inputs to different dendritic regions of a cell results in an integrated output that is relevant to the behavior of the animal. These anatomical relationships are of special interest

\footnotetext{
Received Sept. 25, 1985; revised Jan. 23, 1986; accepted Feb. 14, 1986.

This research was supported by NSF Grants BNS 82-02416 (J.P.M.) and BNS 81-19799 (R.K.M.).

Correspondence should be addressed to Dr. Gwen Jacobs at the above address. Copyright (C) 1986 Society for Neuroscience $0270-6474 / 86 / 082298-14 \$ 02.00 / 0$
}

in sensory systems where primary sensory neurons form a "topographic map" of their receptive fields within the CNS. For example, visual space, auditory space, and body representation are all "mapped" on to the CNS with specific coordinate systems. In the cricket cercal afferent system, wind direction with respect to the animal's body is mapped functionally in the terminal abdominal ganglion. The function of the sensory interneurons in all these systems is to "interpret" these maps of sensory space. This functional interpretation is dependent on neuronal structure at 2 different levels. At a "macroscopic" level, the number and position of a neuron's dendritic branches determine the afferent inputs it receives. At a more microscopic level, the fine structure of the individual dendritic branches determines how synaptic inputs and endogenously generated currents are integrated and transformed into the signals the neuron transmits to other cells. The focus of the work presented here is the examination of the structure/function relationships of an identified wind-sensitive sensory interneuron in the cricket CNS.

The primary afferent input to this interneuron is from sensory neurons associated with filiform hairs on the cerci (Edwards and Palka, 1974) located at the end of the abdomen of the cricket. Each filiform hair is innervated by a single sensory neuron (Gnatzy and Tautz, 1980) that fires a burst of action potentials when the hair is deflected in its socket (Palka et al., 1977; Tobias and Murphey, 1979). The afferents have been divided into 4 groups based on their directional sensitivity, and each class of sensory afferent terminates in a different region of the cercal glomerulus in the terminal ganglion (Bacon and Murphey, 1984). By focusing on these 4 main afferent classes, the cercal glomerulus can be represented as being divided into functionally distinct regions representing 4 different wind directions (Bacon and Murphey, 1984). There is good evidence that the sensory afferents provide monosynaptic excitatory input to the wind-sensitive interneurons (Bacon and Murphey, 1984; Callec et al., 1971; Shepherd and Murphey, in press).

The spatial organization of the afferent terminals has important functional consequences for the wind-sensitive interneurons. An interneuron with dendrites located in one or more of the neuropil regions could receive input from afferents sensitive to those respective wind directions, the sum of which would then represent the "receptive field" of the interneuron. This model was first tested by Bacon and Murphey (1984) and good qualitative agreement was obtained between the receptive fields of the interneurons and the anatomical predictions. However, it is important to distinguish between the overall receptive field of a neuron and its precise "directional sensitivity," which is its differential responsiveness to wind stimuli from different directions within its receptive field. Although the model of Bacon and Murphey (1984) adequately accounts for the receptive fields of some of the identified interneurons, it cannot explain why an interneuron is much more responsive to a narrow range of wind orientations within its broad receptive field (Jacobs and 
Miller, 1985). The experiments described in this paper were designed to elucidate the mechanisms by which the precise directional sensitivity of one particular interneuron results from the integration and transformation of synaptic inputs from different regions of its receptive field. Two factors significantly influence the integration of excitatory inputs within the cell. First, inhibitory inputs have been shown to "sharpen" the directional sensitivity of the cell (Levine and Murphey, 1980a, b). The inhibitory pathway has been shown to be polysynaptic, on the basis of the long and variable latency of the IPSP (Levine and Murphey, 1980b). In these earlier studies, however, sound stimuli were used to stimulate the afferents. Unlike wind, sound is bidirectional and can only distinguish between 2 of the 4 classes of afferents. This has led to some misconceptions regarding the source of both excitatory and inhibitory inputs to the interneuron. In this report, we present the results of experiments using wind, a unidirectional stimulus, to activate the afferents. These experiments characterize the orientations from which wind stimuli activate inhibitory inputs, and demonstrate how these inhibitory inputs affect directional sensitivity.

A second factor that contributes to synaptic integration in the interneuron is its "electroanatomy." The efficacy with which a particular synaptic input can influence the output of a neuron is determined by the attenuation of the PSP between the input and the spike-initiating zone (SIZ). The location of the SIZ in interneuron 10-3, and the location of the 3 different input branches with respect to it, are therefore very important determinants of the cell's directional sensitivity. Using the laser photoinactivation technique to produce lesions at specific sites along the major neurite of 10-3, we have located the probable SIZ of this cell. On the basis of this knowledge, the relationship between the activity recorded in the cell body and the spiking output from the SIZ is explained, and a qualitative model of the cellular basis of directional sensitivity is presented. Preliminary reports of portions of this work have been presented elsewhere (Jacobs and Miller, 1985; Miller and Jacobs, 1984).

\section{Materials and Methods}

Experiments were performed on adult female crickets, Acheta domesticus, 3-24 hr after their final molt. Each cricket was mounted on a Sylgard block that was glued to a Plexiglas platform. Calibrated airstreams were delivered to the cerci of the cricket through a laminar flow nozzle. House air was supplied to the nozzle through a pressure regulator, and controlled with a solenoid-driven valve. For each experiment, the pressure was adjusted while the filiform hairs were observed through the dissecting microscope, and was set to a level that caused an immediate, stable deflection of the hairs, with no observable rebound or vibration. In control experiments ( $n=5$, data not shown), at least 3 different directional plots were recorded from the same cell, each produced using a different pressure. The pressures were one-half, one, and two times the pressure used in subsequent experiments. For these different plots, although the absolute values of the responses at any particular location varied with wind velocity, the normalized polar plots showed no significant variability. The nozzle was mounted on a freestanding carousel that could be rotated around the cricket 360 degrees in the horizontal plane. To measure the directional sensitivity of the interneuron, recordings were obtained in response to wind puffs at 11 positions at $30^{\circ}$ intervals around the animal. The nozzle was aligned with a protractor affixed to the platform to support the cricket in order to insure consistency in stimulus orientation. The recorded response of the neuron to a given stimulus was quantified by measuring the area under the waveform directly from a Nicolet digital oscilloscope, using a standard program. Both inhibitory and excitatory responses could be measured in this way. Each directional sensitivity measurement was repeated at least 3 times in all experiments.

\section{Intracellular recording}

For routine intracellular recording, the terminal ganglion was exposed (Matsumoto and Murphey, 1977) and stabilized in the body cavity with a small steel platform, and bathed in physiological saline (U'Shea and Adams, 1981). Microelectrodes were filled with $2 \mathrm{M}$ potassium acetate and had resistances between 20-40 M . For photoinactivation experiments, the tips of electrodes were filled with 3\% 6-carboxyfluorescein (Kodak) in distilled water and the shanks filled with $2 \mathrm{M}$ potassium acetate. These electrodes were beveled to a resistance of $40-60 \mathrm{M} \Omega$. The electrodes in all experiments were coated with silver paint to within 1 $\mathrm{mm}$ of the tip. The silver paint was connected to the driven shield in the headstage of the amplifier. For most experiments, signals were rccorded with a Getting amplifier, displayed on a Nicolet digital oscilloscope and stored on floppy disks. For photoinactivation experiments, a single electrode voltage clamp (SEC) was used in current clamp mode for injecting current and recording voltage simultaneously. This was used to determine the current-voltage relationship of the cell and to determine the apparent reversal potential for synaptic potentials.

\section{Anatomy}

The interneurons were injected with $100 \mathrm{~mm}$ hexaminocobaltic chloride (Scientific Products) by applying constant $+3 \mathrm{nA}$ current for $10 \mathrm{~min}$ The preparation was stored at $4^{\circ} \mathrm{C}$ for $1 \mathrm{hr}$ to allow diffusion, and processed for silver intensification of cobalt (Bacon and Altman, 1977; Murphey et al., 1980). Wholemounts were mounted in a mixture of Canada balsam and xylene and drawn at $400 \times$ with a camera lucida affixed to a Zeiss microscope. The stereo pair photograph was made using the technique of Tieman et al. (in press).

\section{Photoinactivation of individual dendrites}

The technique used in these experiments was a modification of the one developed by Miller and Selverston (1979) for killing whole cells in the stomatogastric ganglion and is described in detail elsewhere (Jacobs and Miller, 1985; Kater et al., 1986). The light source was a $10 \mathrm{~mW}$ heliumcadmium laser (Liconix) directed through the optics of a stereo dissecting microscope (Wild M5APO) using a specially designed epifluorescence attachment. The microbeam could be focused to a spot approximately $30 \mu \mathrm{m}$ in diameter. The laser microscope was mounted on a platform that could be moved independently of the preparation so that the microbeam could be moved to any region of the ganglion, and thus localized to any part of a dye-filled neuron. The beam was aligned with a graticule in the eyepiece of the microscope and thus could be positioned to the appropriate location on the preparation without actually illuminating the cell.

The photoinactivation procedure was as follows: Signals were recorded with a SEC in current clamp mode at a cycle frequency of 2-3 $\mathrm{kHz}$. The headstage of the SEC was constantly monitored with a dedicated oscilloscope to insure critical capacitive compensation. First, fluorescein was injected iontophoretically, using a steady $-3 \mathrm{nA}$ current for approximately $5 \mathrm{~min}$, or until the cell body appeared yellow/green. The preparation was shielded from room light to protect against nonspecific photoinactivation damage. Then the current/voltage $(I / V)$ relationship was measured using the hyperpolarizing half-cycle of a 0.25 $\mathrm{Hz}$ sine wave ( $-2 \mathrm{nA}$ maximum amplitude) from a waveform generator. Responses to wind stimuli from 11 different directions were then recorded. Next, a single dendrite was illuminated with the laser, using multiple exposurcs $1-2 \mathrm{sec}$ in duration. If the cell was illuminated for a period longer than $5 \mathrm{sec}$, it would begin spiking rapidly, the spikes would broaden, and the cell would steadily depolarize to ground, indicating irreversible damage to the entire cell. This was a frequent cause of failure in these experiments. The success rate was approximately 1 in 10 attempts. By using short periods of illumination and monitoring the spontaneous activity of the cell, the photoinactivation could be limited to a specific region without damaging the rest of the cell. In most experiments, the cell depolarized as much as $5 \mathrm{mV} 1$ to $2 \mathrm{~min}$ after the illumination, but repolarized within $15 \mathrm{~min}$. After the cell stabilized, a second I/V measurement was taken to check for nonspecific damage. If the input resistance was decreased with respect to the preillumination control, indicating a current shunt, the results were discarded. The criteria used to determine acceptable data were (1) an increase in input resistance to a new stable value after the photoinactivation and (2) no apparent change in the resting membrane potential at the end of the experiment.

All experiments reported in this work were repeated at least 3 times, with similar results. 
Figure 1. Interneuron 10-3. Stereomicrograph of a wholemount preparation stained with hexaminocobaltic chloride and silver-intensified. Each of the 3 distinct dendritic regions has been labeled $X, Y$, or $Z$. (Stereogram was prepared by Dr. David Tieman.)
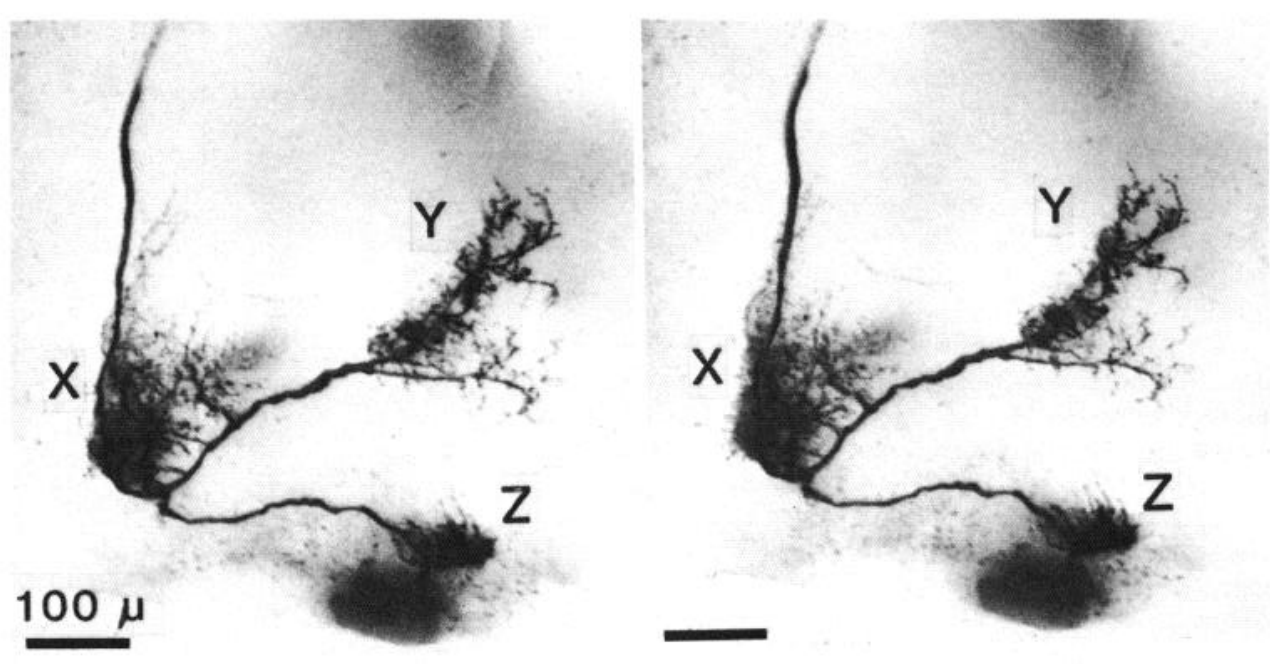

\section{Results}

\section{Anatomy of interneuron 10-3}

In the terminal abdominal ganglion there are two 10-3 interneurons, each a mirror-symmetric homolog of the other. Each interneuron has 3 dendrites, each located in different, nonoverlapping regions of the terminal ganglion. To simplify the presentation of the results, the 3 dendrites have been designated X, Y, and Z (Fig. 1). The arborization of these dendritic branches in different neuropil regions with respect to the topographic "map" of afferent terminals has important functional consequences for the interneuron. A puff of wind from a particular direction deflects a specific subset of the filiform hairs to its optimal stimulus orientation and consequently stimulates the associated subset of sensory afferents. Wind blown at the cerci from another direction will activate a different subset of afferents. Therefore, at the level of the sensory afferents, the direction of a particular wind stimulus is encoded by the specific populations of afferents that respond to that stimulus. Wind direction is encoded anatomically in the CNS by the segregation of afferent terminals into different local regions.

When the locations of the 3 dendrites of interneuron 10-3 were compared with the 4 regions of afferent termination described by Bacon and Murphey (1984, Fig. 9), there were clear areas of overlap between the dendrites and selected classes of afferents. Dendrite X was located in a neuropil region occupied primarily by afferents that respond to wind directed at the lateral face of the left cercus, the lateral " $T$ " hairs. Dendrite $Y$ overlapped with afferents from the right cercus stimulated by wind directed at the front of the animal, the anterior "L" hairs. Dendrite $\mathrm{Z}$ overlapped with afferents stimulated by wind directed at the medial face of the right cercus, the medial $T$ hairs. The remaining class of afferents, the posterior $L$ hairs, had virtually no overlap with this interneuron. Likewise, medial $\mathrm{T}$ and anterior $\mathrm{L}$ hairs on the cercus ipsilateral to the axon, and lateral $T$ hairs ipsilateral to the soma, did not overlap with the cell.

The anatomy suggests that each of 10-3's dendrites should receive input from a different class of afferents. The summation of these 3 inputs must contribute to the response properties of the neuron.

\section{Directional sensitivity of 10-3}

The response properties of interneuron 10-3 to wind stimuli from different orientations were recorded intracellularly from the cell body $(n=45)$ and plotted in polar coordinates (Fig. 2). The response of 10-3 to a wind puff from an optimal stimulus orientation was composed of a depolarization and a burst of action potentials that was maintained for the duration of the stimulus (Fig. 2, 2). The interneuron was maximally sensitive to wind from the left side of the animal's body. The response was greatly reduced in amplitude on the right side and inhibited by wind directed at the right rear.

As a first step in relating each response to selective activation of a different set of afferents, the movements of the hairs on the cerci were observed at each stimulus orientation. By observing which of the 4 classes of hairs were deflected by a stimulus from a particular orientation, the anatomical map discussed above (Bacon and Murphey, 1984) was used to predict which of the 3 dendrites of 10-3 should be activated by the stimulus. This is indicated by the letters $\mathrm{X}, \mathrm{Y}$, and $\mathrm{Z}$ around the perimeter of the polar plot (Fig. 2). These observations were used to compare the observed activity in the interneuron to activation of different classes of hairs presumed to provide input to each dendrite.

The maximal responses in neuron 10-3 occurred at positions that should primarily activate dendrites $X$ and $Z$. At positions that should activate dendrite $Y$ alone (5 and 6 ), the cell fired at a much lower frequency. The response properties of the interneuron demonstrated in Figure 2 fit the anatomical predictions for the receptive field reasonably well. However, the maximal directional sensitivity occurred when afferents overlapping with 2 of the 3 dendrites were activated, and the response decreased in magnitude at other positions. At some positions, however, the cell was strongly inhibited by the wind stimulus (Fig. 2, 4). These positions activated sets of afferents that do not overlap anatomically with interneuron 10-3 and thus may have been activating a polysynaptic inhibitory pathway.

These results suggest that the anatomy of the interneuron can be used only to predict its excitatory receptive field, i.e., the orientations from which wind stimuli will evoke an excitatory response. Dendritic anatomy cannot be used to predict the origin or efficacy of inhibitory inputs, nor can it be used to predict the relative efficacies of stimuli from different orientations within the receptive field. However, the net response of the cell must be due to summation of excitatory and inhibitory inputs from both cerci, and the response to inputs from each cercus should be separable and specific to particular dendrites. The experiments in the following sections were designed to characterize the response of 10-3 in terms of excitatory and inhibitory inputs to individual dendrites.

\section{Excitatory receptive fields of individual dendrites}

The anatomical data suggest that each of 10-3's dendrites overlaps with a separate class of afferent, sensitive to a different wind 


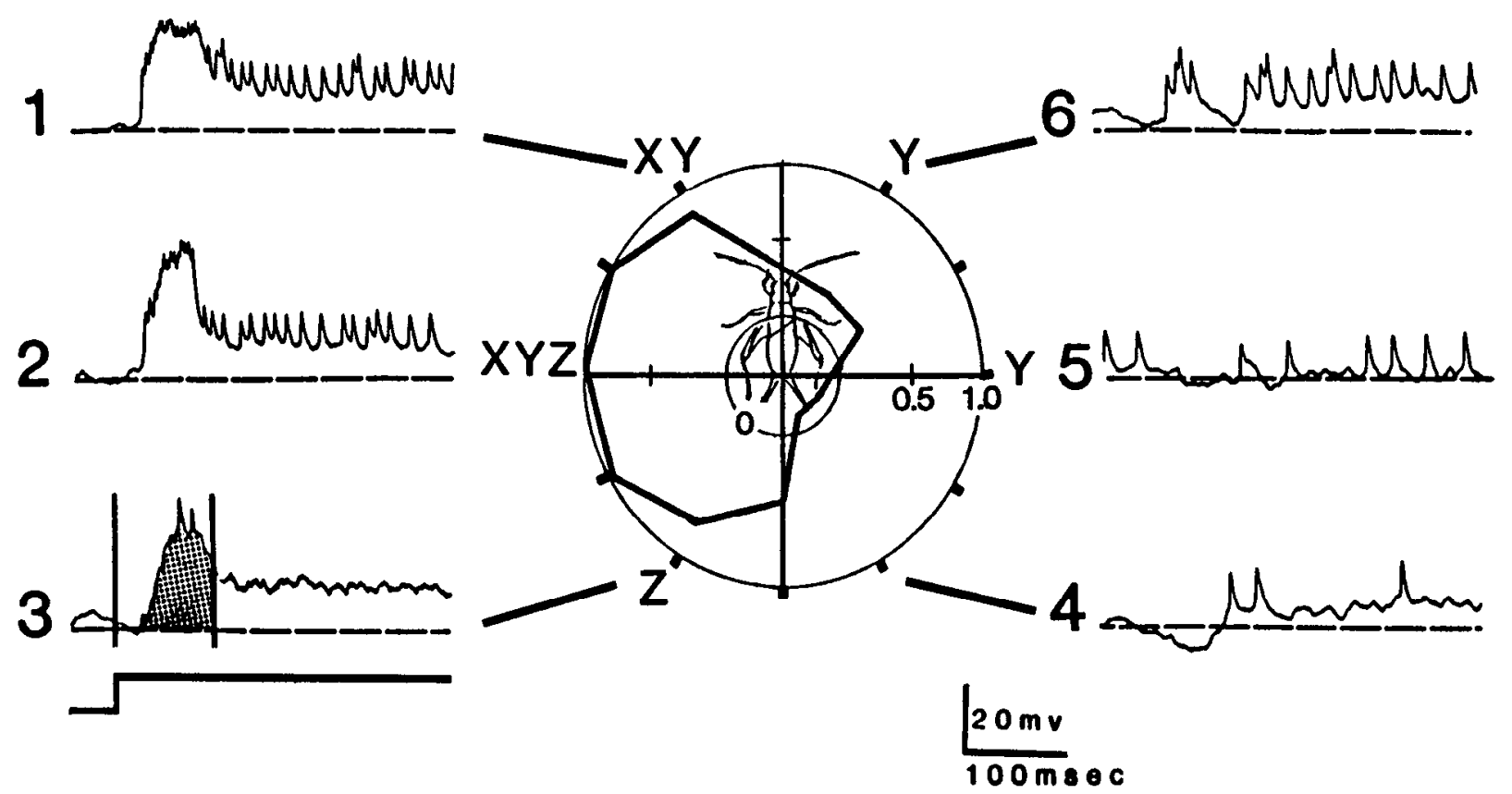

Figure 2. Directional sensitivity of interneuron 10-3. Polar plot of the directional sensitivity is shown in the center, surrounded by examples of intracellularly recorded responses from 6 different stimulus orientations. Response amplitude at each wind orientation on the polar plot was measured by integrating the area under the intracellularly recorded response for the first $100 \mathrm{msec}$ following stimulus onset. For example, the area measured from trace 3 is shown shaded. Areas for all orientations were scaled to the maximum area value and plotted as a function of stimulus orientation. Outer circle, 1.0, represents maximum area value for this experiment. Inner Circle is labeled 0 , and values inside this circle represent inhibition of 10-3, i.e., a net hyperpolarization below the resting level. These conventions are the same in all subsequent directional sensitivity plots. The letters at each stimulus orientation refer to activation of afferent populations presumed to provide excitatory input to dendrites $X, Y$, and $Z$ (see Fig. 4).

direction. If this is true, and if single classes of afferents could be isolated and stimulated selectively, then the resulting response of 10-3 should be different for each of these classes. The following experiments were designed to test this prediction by reducing the afferent input in each case to a single class of hairs.

For comparison with the following experiments, the directional responses of 4 different afferents, located on the right cercus, were obtained by recording intracellularly in their axons (superimposed in Fig. 3). These afferents represent the 4 main groups described by Bacon and Murphey (1984). There was considerable overlap between the response curves for the medial $\mathrm{T}(n=4)$ and posterior $\mathrm{L}(n=3)$ afferents, and between the curves for lateral $\mathrm{T}(n=4)$ and anterior $\mathrm{L}(n=4)$ afferents. Among afferents from the same class (not shown), the curves overlapped to a much greater extent.

The hairs associated with these different classes of afferents have been mapped on the cercus in detail by Bacon and Murphey (1984) and more recently by Walthall and Murphey (1986). Using these maps, we isolated small patches of 4 to 6 hairs on one cercus; all other hairs were covered with Vaseline (see Matsumoto and Murphey, 1977, for methods). In all experiments, the cercus contralateral to the one with the isolated patch was left uncovered long enough to identify the interneuron, and then covered with Vaseline, leaving only those hairs in the patch free to move. After the sensitivity of the interneuron to this reduced afferent input was measured, the hairs in the patch were plucked. If a response could still be recorded from 10-3 after all hairs were removed, the experiment was discarded.

The results of 3 such experiments are shown in Figure 4. When the patch of intact hairs consisted of lateral $T$ hairs on the left cercus (ipsilateral to the axon), the response of the cell was maximal for wind directed at the lateral face of the left cercus $(n=4)$ (Fig. 4A). At other positions the response was negligible. When the patch consisted of 4 anterior $L$ hairs on the right cercus (ipsilateral to the soma), the response of the cell was maximal to wind blown at the front and right side of the animal's body $(n=4)$ (Fig. $4 B)$. Wind from the rear evoked no excitatory response from the cell. Figure $4 C$ demonstrates the response of the interneuron to 4 medial $T$ hairs on the cercus ipsilateral to the soma $(n=4)$. Note that the response of the cell was maximal to wind blown at the medial face of the cercus. Thus, in each case the receptive field of 10-3 matched the receptive field of the hairs in the patch. Note that the receptive fields for individual dendrites, as shown in Figure 4, are larger than the receptive fields for individual afferents shown in Figure 3. This indicates that the receptive field of each dendrite is made up of

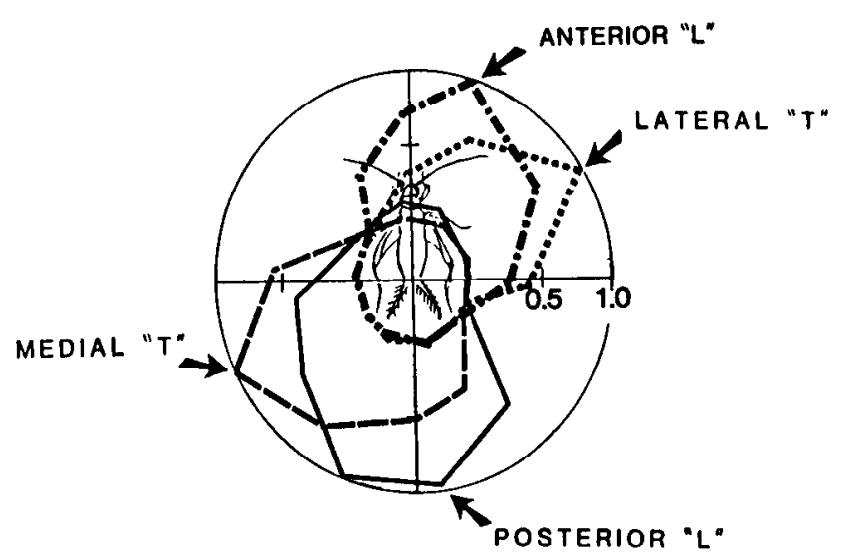

Figure 3. Comparison of the directional sensitivites of 4 different filiform afferents located on the right cercus. Each afferent represents a different functional class and has a distinct directional sensitivity to wind stimuli. Coordinate system, see Figure 2. 
A

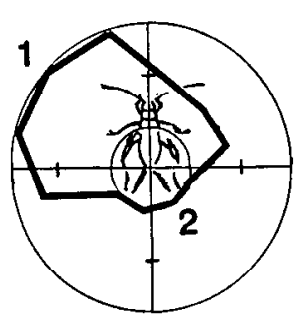

B

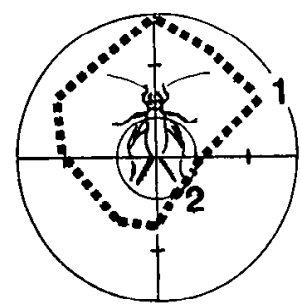

C

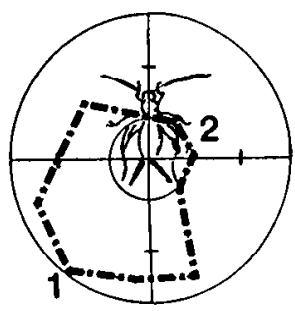

LATERAL " $T$ " (DENDRITE $X$ )
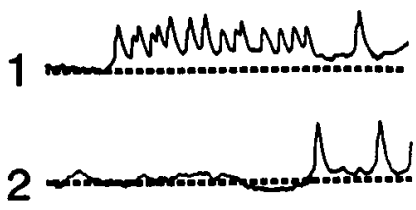

\section{ANTERIOR " $L$ "} (DENDRITE $Y$ )

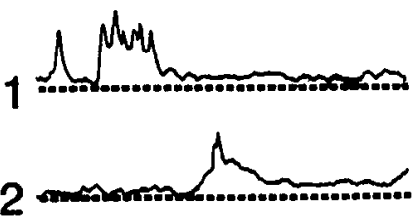

\section{MEDIAL "T" (DENDRITE Z)}
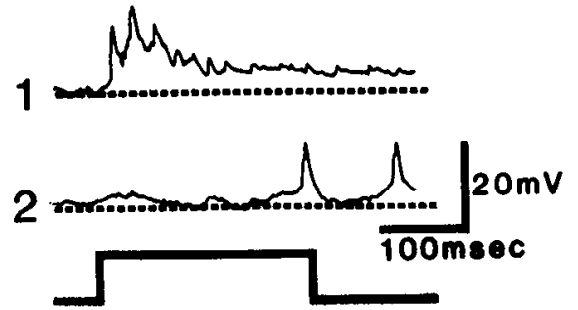
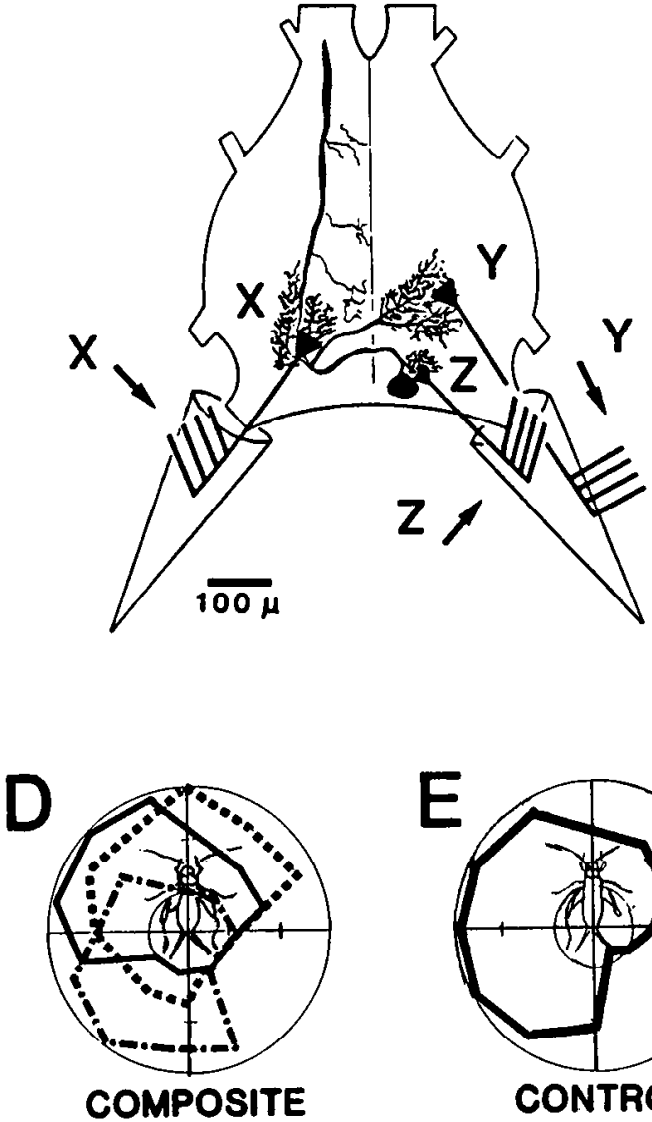

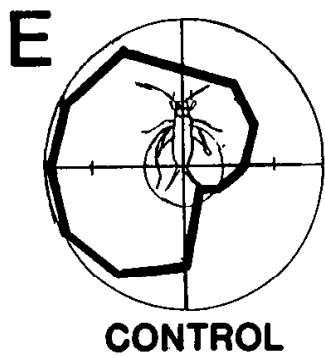

Figure 4. Directional senstivities of individual dendrites of 10-3. $A$, Response of 10-3 to wind stimuli with the afferent input reduced to 4 lateral "T" hairs on the cercus contralateral to the soma. Recordings from maximal and minimal positions, records 1 and 2 , respectively. $B$, Same as $A$, except with afferent input reduced to 4 anterior " $L$ " hairs on the cercus ipsilateral to the soma. $C$, Same as $A$, except with afferent input reduced to 4 medial "T" hairs on the cercus ipsilateral to the soma. $D$, Superposition of curves from $A, B$. and $C$. $E$, A control in which directional sensitivity was measured with both cerci intact.

a combination of the receptive fields of all of the afferents that synapse with it.

The directional curves for all 3 excitatory patches have been superimposed and compared to a curve from a control experiment in which both cerci were intact (Fig. $4, D, E$ ). It is apparent that the interneuron tested with each patch in isolation was maximally sensitive at different wind positions. In addition, there were considerable overlaps between the dircctional curves. When the composite plot (Fig. $4 D$ ) was compared with a control (Fig. $4 E$ ) in which both cerci were intact, the range of sensitivity of the 3 curves (i.e., the receptive field) matched that of the control.

No other classes of hairs cvoked an excitatory response in 103. However, 2 classes of hairs, lateral $T$ hairs on the cercus ipsilateral to the soma and posterior L hairs on either cercus, evoked an inhibitory response in interneuron 10-3. The nature and significance of these inhibitory inputs will be considered in detail below.

\section{Photoinactivation of individual dendrites}

The experiments described above provide strong correlative evidence that the excitatory input to $10-3$ is mediated by the 3 classes of hairs that overlap anatomically with its 3 dendrites. They also suggest that each dendrite might exhibit a unique directional sensitivity. To definitively test for this possibility, it was necessary to isolate one dendrite from the rest of the neuron in situ. This was accomplished by selective photoinactivation experiments using a laser microbeam. In one set of photoinactivation experiments, dendrite $\mathrm{Y}$ was ablated, and the subsequent effects upon directional sensitivity observed $(n=3)$. In order to simplify this experiment, the cercus contralateral to the soma was covered with Vaseline in order to block the inputs to dendrite $X$. The results of this experiment are shown in Figure $5 C$. The outer solid curve represents the directional sensitivity to inputs onto dendrites $\mathrm{Y}$ and $\mathrm{Z}$ from the cercus ipsilateral to the soma, before photoablation. After illuminating dendrite $Y$, the cell was allowed to stabilize for $15 \mathrm{~min}$, and its "new" directional sensitivity was measured and plotted as the dashed curve. Both curves in Figure $5 C$ were scaled to their own maximum values. The response of $10-3$ was most sensitive to wind directed at the medial face of the right cercus. An example of the response before and after the photoinactivation is shown at 3 positions on the curve. Wind directed at the front of the animal at position 3 , which is an optimal stimulus for dendrite $Y$, failed to elicit an excitatory response as it had in the control. The response recorded after photoinactivation appeared to be weakly inhibitory. This experiment demonstrates that the $\mathrm{Y}$ dendrite receives input from a select class of afferents, the anterior $L$ hairs, that respond to wind directed at the front of the animal.

In a second set of photoinactivation experiments, dendrite $Z$ was isolated from the neuron by illuminating the region indicated with an asterisk in Figure $5 A$ (Jacobs and Miller, 1985). Under these conditions, the directional sensitivity of the lasertransected cell was changed: the cell was maximally sensitive to 

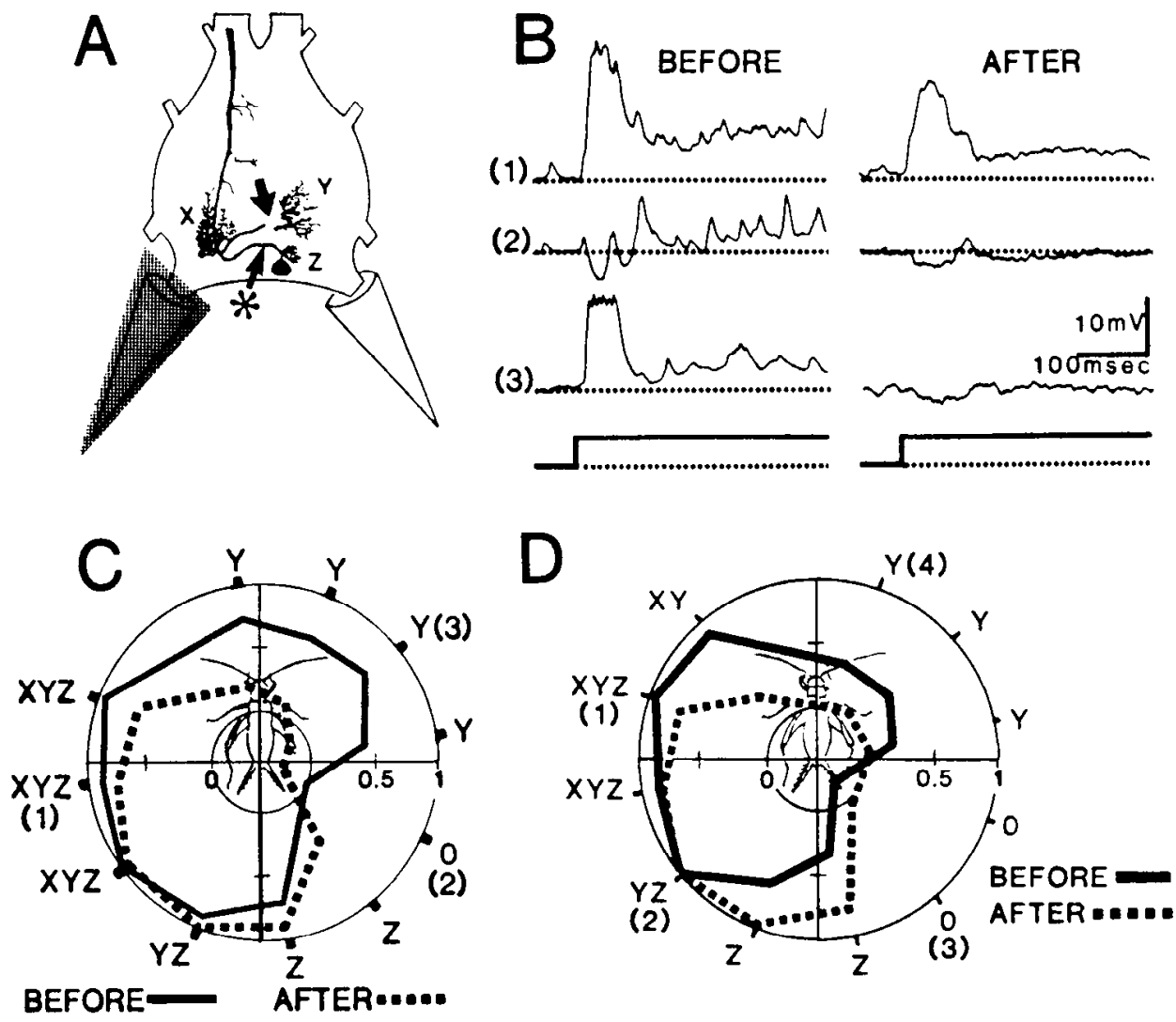

\begin{abstract}
Figure 5. Photoinactivation of dendrite $Y$ in a preparation with left cercus covered with Vaseline. A, Arrow on the drawing of interneuron 10-3 indicates site of illumination. Asterisk indicates site of illumination for the isolation of dendrite $Z$ from a different experiment. $B$, Sample responses are shown at 3 different stimulus orientations before and after photoinactivation. Bottom traces indicate wind stimuli. $C$, Directional sensitivity plots of the response of 10-3 to wind stimuli to the cercus ipsilateral to the soma before (solid line) and after (dashed line) dendrite $Y$ was illuminated with the laser. Both directional plots were scaled to their own maximum values. $D$, Directional sensitivity plots of interneuron 10-3 measured before and after dendrite $Z$ was isolated from the rest of the neuron by photoinactivation at the site in $A$ indicated with an asterisk (Jacobs and Miller, 1985).
\end{abstract}

wind blown at the medial face of the right cercus, the optimal stimulus for the afferents that overlap with dendrite $Z$ (Fig. $5 D$ ). Thus, by isolating dendrite $\mathrm{Z}$ and the soma from the rest of the neuron with the photoinactivation technique, we determined that this dendrite receives excitatory input from medial $T$ afferents. In addition, it receives no visible inhibitory inputs and is located proximal to the spike-initiating zone of the neuron.

The directional sensitivities of interneuron 10-3 after these 2 different photoinactivation experiments were very similar (compare dotted lines in Fig. 5, $C$, and $D$ ). In each experiment, the directional sensitivity of dendrite $Z$ was revealed: in the first, by isolating dendrite $Z$ from the rest of the dendrites; in the second, by mechanically blocking the input to dendrite $X$ and photoinactivating dendrite $\mathrm{Y}$.

\section{Inhibitory inputs to interneuron 10-3}

Inhibitory inputs have been shown to affect the directional sensitivity of many of the identified interneurons in this system (Levine and Murphey, 1980a, b; Matsumoto and Murphey, 1977). In particular, the effects of inhibitory inputs to interneuron 10-3 were demonstrated as effectively sharpening the directional sensitivity of this cell. To characterize the mechanisms by which this "sharpening" is achieved, it was necessary to determine which stimulus orientations were most effective in eliciting inhibitory responses in the interneuron. Cell body recordings from interneuron $10-3$ in an animal with both cerci intact revealed that wind stimuli aimed at the rear of the cercus ipsilateral to the soma resulted in an inhibitory response ( $n=$ 45) (Fig. 6, $A, B$ ). At this orientation, the neuron was hyperpolarized (as indicated on the polar plot by points within the inner circle).

In order to determine the source of the inhibitory input, the response of interneuron 10-3 to inputs from each cercus was recorded independently and compared to the response with both cerci intact. To do so, we initially recorded the responses of 10-3 with both cerci intact, and then with one of the cerci covered with Vaseline. Covering one of the cerci with Vaseline silences all of the afferents that project to 10-3 in the respective hemiganglion. The other $10 \%$ of the afferents that cross the midline do not overlap anatomically with interneuron 10-3 (Murphey and Lemere, 1984). When the cercus ipsilateral to the soma was blocked, there was a reduced sensitivity response of the interneuron to wind, from some orientations more severely than from others $(n=8)$ (Fig. $6 A$, dotted line). The maximum response still occurred at the stimulus orientation that should stimulate lateral $\mathrm{T}$ hairs overlapping with dendrite $\mathrm{X}$ (Fig. 6A,1). The response decreased abruptly at the rear of the animal (2) and was inhibited at stimulus orientations (3) around the right side of the animal's body. Note that the response measured at position 2 in the control was excitatory with both cerci intact and inhibitory after the cercus was covered. This indicates a co-activation of excitatory and inhibitory inputs at this stimulus orientation with both cerci intact.

When the cercus contralateral to the soma was covered (Fig. $6 \mathrm{~B}$, dotted line), the directional sensitivity of the neuron was also reduced in area and was quite different in shape, as compared to both the control (solid line) and the response to inputs to the left cercus alone (dotted line) $(n=13)$. With only the right cercus intact (dotted line), the interneuron was sensitive to inputs activated by wind from the front (1) and the left rear (2) of the animal, and inhibited by inputs from the right rear (3). In this experiment, the dotted curve representing excitatory inputs to dendrites $\mathrm{Y}$ and $\mathrm{Z}$ extended outside the area of the control curve at the rear of the animal (2). This suggests that excitatory inputs from the right cercus are normally masked by co-activation of inhibitory inputs from the left cercus at these wind positions (Edwards and Palka, 1974). It appears that inhibitory inputs were maximally activated in both experiments by wind blown at the rear of each cercus ( 3 on each plot). This 

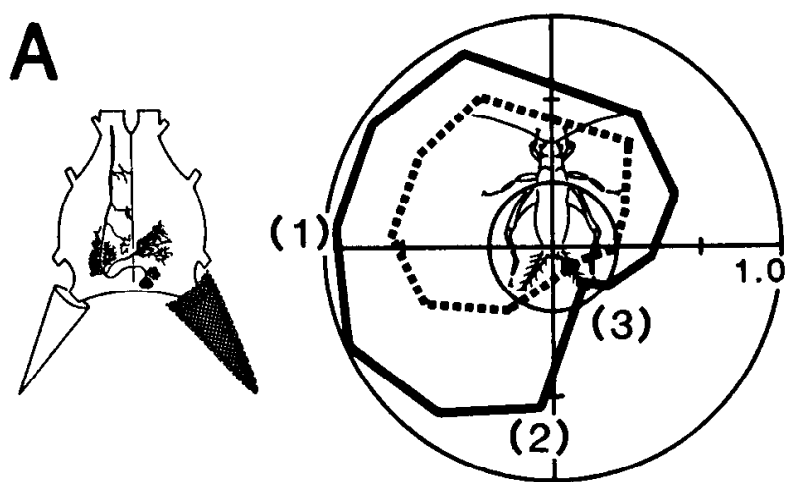

BOTH CERCI
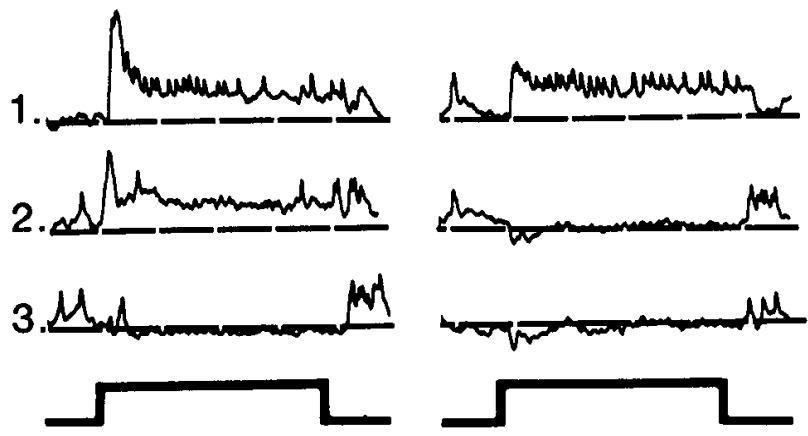
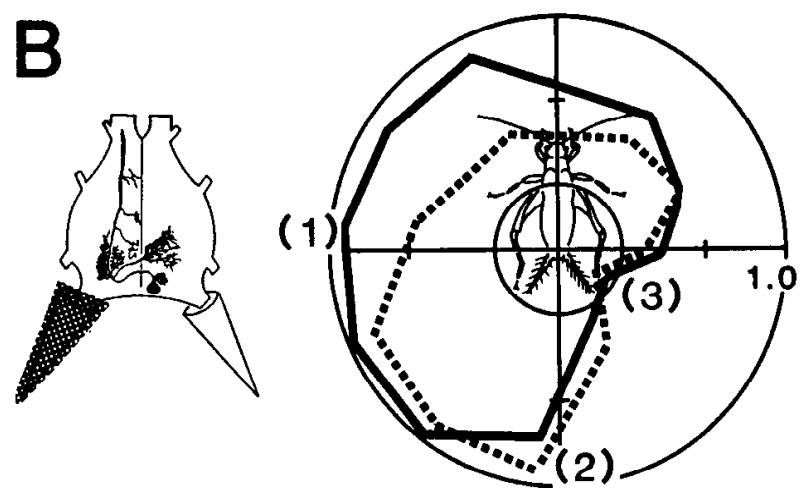

BOTH CERCI
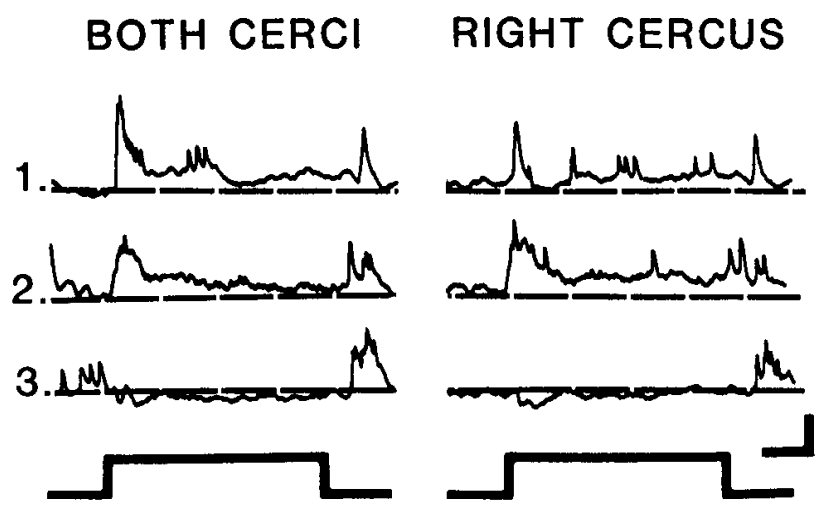

Figure 6. Activation of inhibitory inputs to interneuron 10-3 by wind stimuli. $A$, Directional sensitivity plot of interneuron $10-3$ to inputs from both cerci was measured and plotted in the solid line. After covering the cercus ipsilateral to the soma (see drawing, left), directional sensitivity was measured again, scaled to the control curve, and plotted (dashed line). Sample responses recorded for 3 different positions (indicated by numbers on the polar plot) are shown below the plot for inputs from both cerci and for the remaining left cercus. $B$, Complementary experiment, in which the cercus contralateral to the soma was covered (drawing, left). Solid line indicates responses to inputs from both cerci; dashed line (scaled to the control) represents responses to inputs from the ipsilateral cercus.

result suggests that inhibitory inputs to this interneuron can be activated by afferents on either cercus. The inhibitory inputs have 2 effects on this cell, depending on the stimulus orientation from which they are activated. At some orientations, inhibition can completely block spiking activity (Fig. 6, $A, B, 3$ ). At other orientations it can suppress some of the activity evoked by excitatory inputs coactivated by the stimulus (Fig. $6, A, B, 2$ ).

Since it was quite difficult to determine whether or not a particular wind stimulus activated only inhibitory inputs, small patches of hairs were isolated and stimulated independently in order to identify the class of afferents responsible for activating inhibition. As is shown in Figure 7, 3 populations of afferents activated inhibitory input to 10-3: posterior $\mathrm{L}$ hairs on both cerci $(n=12)$ (Fig. $7 A)$, and lateral T hairs on the cercus ipsilateral to the soma $(n=4)$ (Fig. $7 B)$. None of these hair classes overlaps anatomically with $10-3$, and therefore must be stimulating an inhibitory interneuron or interneurons with input dendrites in these neuropil regions.

\section{Summation of inputs}

The data indicate that the directional sensitivity of 10-3 results from a summation of both excitatory and inhibitory inputs from both cerci. To assess the degree of nonlinearity in the summation of these different inputs at different stimulus orientations, the optimal approach would be to (1) record the response of the neuron to inputs from both cerci, (2) record the response to each cercus independently, and (3) compare the sum of the responses of the 2 single cerci to the control case with both cerci intact. Any regions within which the sum and control curves are not superimposable would indicate a deviation from linearity. Unfortunately, this experiment is impossible, since the only feasible way to block afferent inputs from a cercus is with Vaseline, and this procedure is irreversible. However, by recording from one $10-3$ and its mirror-symmetric contralateral homolog in the same experiment, it was possible to carry out an equivalent experiment $(n=7)$ (Fig. 8). First, the directional sensitivity of the interneuron was measured in an animal with both cerci intact (Fig. 8C). The cercus ipsilateral to the soma was then covered with Vaseline and the responses were measured a second time. These responses represent the contribution of inputs from the cercus contralateral to the soma (Fig. 8A). To obtain a set of responses representing the contribution from the other cercus in the same animal, the contralateral homolog of 10-3 was impaled, and its directional sensitivity to the remaining intact cercus was measured. This directional sensitivity curve was then reflected $180^{\circ}$ around an axis along the center of the animal, so as to represent the complementary inputs to the initial 10-3 from the cercus ipsilateral to the soma (Fig. $8 B$ ). Both curves were scaled to the maximum response recorded in the control. The linear sum of these 2 curves was calculated and superimposed (dashed line) on the original control curve (Fig. 8C, solid line). In general, the directional sensitivity of the summation curve matched that of the control curve. However, the sum was greater than the control at almost all positions. This mismatch can be explained as a result of (1) nonlinear summation of excitatory inputs, and (2) co-activation of excitatory and inhibitory inputs. These points will be considered in detail in the discussion.

\section{Input/output relationship of 10-3}

The information extracted from the sensory input to this interneuron is transferred to thoracic ganglia by patterns of action 


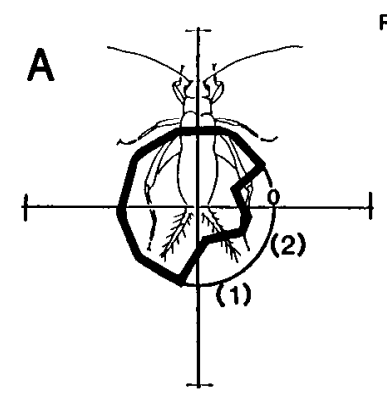

RIGHT CERCUS, POSTERIOR "L" haIRS

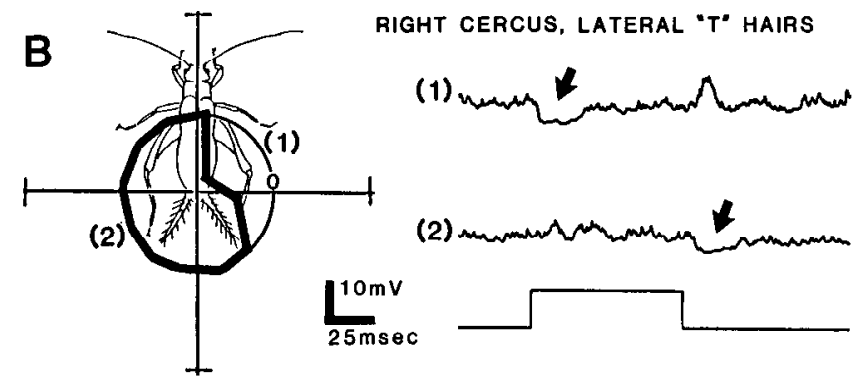

Figure 7. Classes of afferents that activate inhibitory inputs onto 103. $A$, Response of 10-3 to wind stimuli with afferent input reduced to a patch of posterior " $L$ " hairs on the cercus ipsilateral to the soma. No excitatory responses were recorded from any stimulus orientation. Maximal inhibitory response is shown in position 1. At position 2, recorded IPSP was smaller. $B$, Same as $A$, except with afferent input reduced to a patch of lateral " $\mathrm{T}$ " hairs on the cercus ipsilateral to the soma. Position $I$ demonstrates the IPSP evoked by the stimulus in the optimal orientation. At position 2, which is polar opposite to position 1, an IPSP is evoked at the end of the stimulus. Each record is an average of 5 trials.

potentials, which are generated through summation of complex synaptic currents at the SIZ. In the experiments presented in the following section, the factors that influence the integration of inputs at the SIZ were examined.

\section{Location of the spike-initiating zone}

Spontaneous and evoked action potentials, as recorded in the cell body of interneuron $10-3$, appear as small (3-5 mV) tran- sicnts. This implics that the cell body and proximal dendritic membrane are incapable of sustaining a regenerative action potential, owing, presumably, to either a low density or a total lack of active conductance channels. The severe attenuation of the action potentials suggests that the boundary between the "passive" and "active" membrane is locatcd some distance out along the major neurite. In order to determine where the active membrane begins, a series of focal laser ablations were performed. The rationale was that a transection of the dendrite proximal to the active membrane would eliminate action potentials, and that a transection of the dendrite distal to the boundary would not.

For the series of photoablation experiments described previously (Jacobs and Miller, 1985), the neurite was lesioned within the region labeled " 1 " in Figure 9. After such ablations, all spiking activity in the neuron ceased. Neither wind-evoked depolarization nor direct intracellular depolarization could evoke spikes from such cells. Thus, the SIZ must be located at or beyond the intersection of dendrite $Y$ with the main neurite.

In an attempt to locate the SIZ more precisely, the laser was used to irradiate several spots along the axon for periods of 3 $5 \sec (2$ in Fig. 9). There were no observable changes in spike amplitude or duration resulting from these irradiations. This indicates that these ablations were distal to the SIZ. However, when the laser was aimed at the position marked with a short arrow in Figure 9, there was an immediate (within $1 \mathrm{sec}$ ) broadening of the spikes $(n=7)$. Within $1 \mathrm{~min}$, all spiking activity had ceased completely. Thus, there can be no active membrane between the site marked with an arrow and the cell body, and this region must represent the transition between active and passive membrane in the cell. Therefore, the SIZ must lie at or beyond this region of the cell. Since this region is also the site of convergence of all dendritic inputs, it would receive the maximum depolarization from excitatory synaptic inputs. It is thus likely to be the normal site of spike initiation. The precision of this technique was judged to be within approximately $100 \mu \mathrm{m}$, the total area that was visibly deteriorated when a spot of 30 $\mu \mathrm{m}$ diameter was irradiated with the laser.

\section{Summation of inputs at the spike-initiating zone}

An analysis of the input/output characteristics of a neuron requires simultaneous monitoring of subthreshold synaptic input
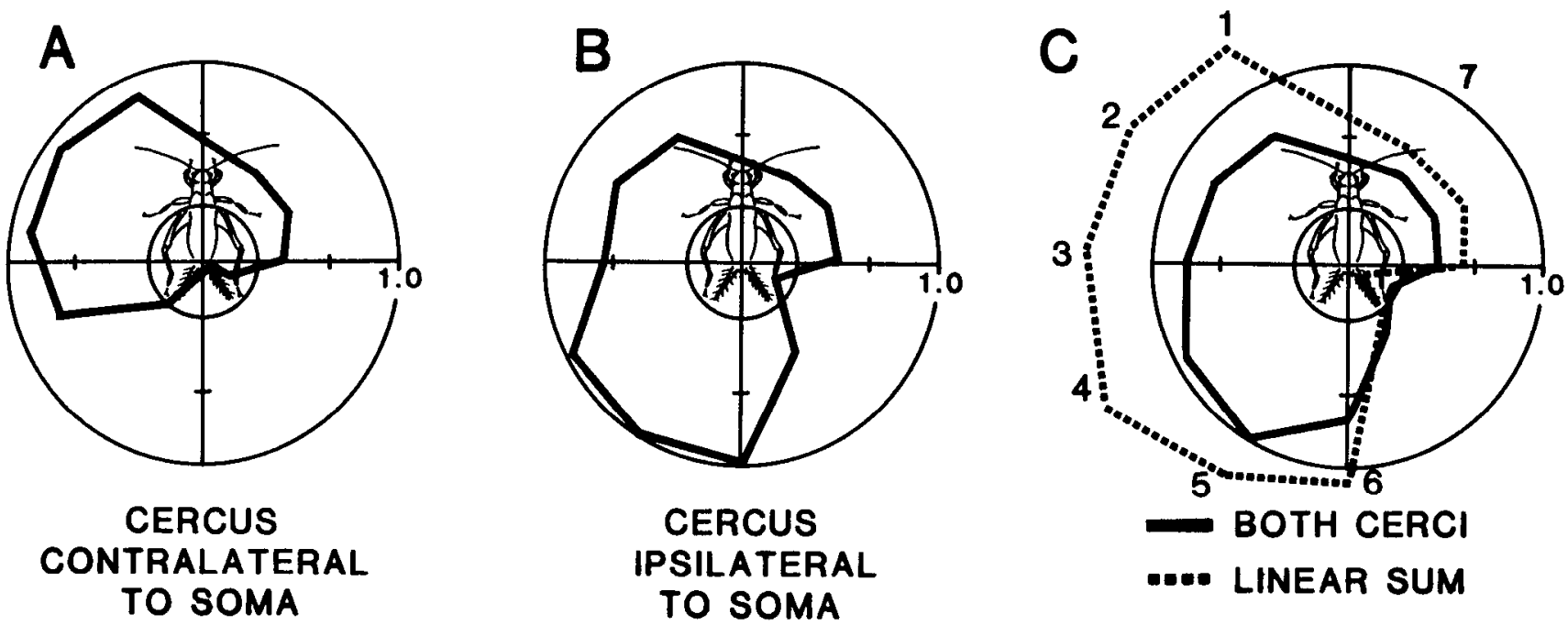

Figure 8. Nonlinearity of summation of inputs from each cercus. A, Directional response of $10-3$ to inputs from the cercus contralateral to the cell body after right cercus was covered with Vaseline. Values for this plot were scaled to the maximum response when both cerci were intact (solid line in $C$ ). $B$, Directional response of the contralateral homolog of 10-3 to the intact right cercus in $A$. These values were also scaled to the maximum response of the control. This curve has been refiected $180^{\circ}$ around the axis of the animal's body. $C$, Solid line is the control directional response recorded with both cerci intact, prior to measuring the response shown in $A$ or $B$. Dashed line represents the linear sum of curves $A$ and $B$. 


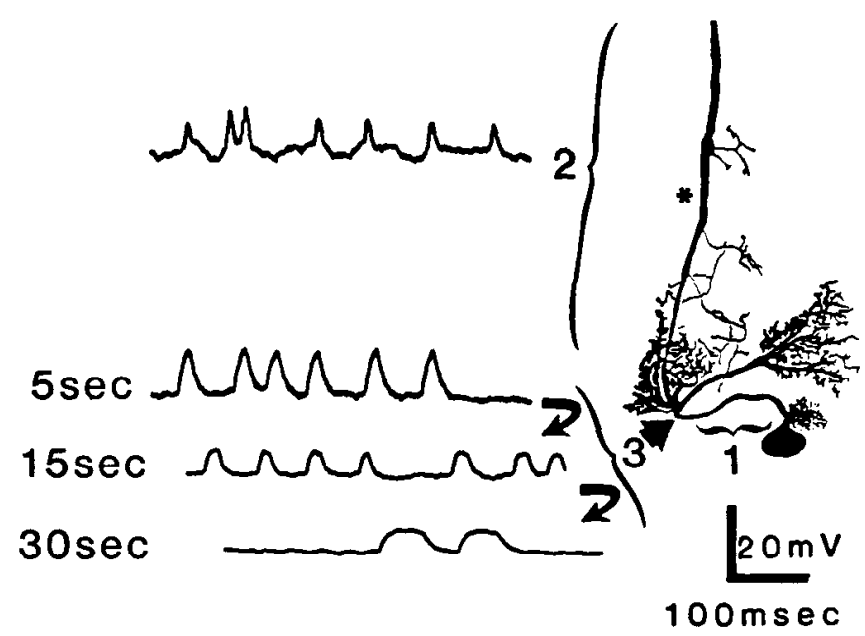

Figure 9. Location of the spike-initiating zone. Records from an experiment in which a laser beam was directed at several different sites on 10-3. When positions within region 2 were illuminated for $5 \mathrm{sec}$, spontaneous activity was unchanged. Top record was taken after the cell was illuminated at the region marked with an asterisk. When the position indicated with the large arrow was illuminated, spikes broadened immediately, and spike-generating ability was lost (lower 3 traces).

and spiking output. Levine and Murphey (1980b) have shown that passively propagated spikes recorded in the cell body of neuron 10-3 occur one-for-one with spikes recorded extracellularly from the axon. Thus, by recording intracellularly from the soma of this neuron, it is possible to monitor synaptic input and spiking output simultaneously.

To measure the input/output relationship of this neuron to wind stimuli, the response properties of the neuron to wind stimuli were measured and evaluated in 2 ways $(n=12)$. The area under each response for the first $100 \mathrm{msec}$ after stimulus onset was measured at each wind position, scaled to the maximum value of all such responses, and plotted in polar coordinates (Fig. 10A, solid line). This plot represents the summed synaptic inputs, and also the receptive field, as monitored at the soma. The action potentials recorded from these records were counted for the same time period, normalized to the maximum value, and superimposed on the same polar plot (Fig. 10 $\mathrm{A}$, dotted line). This curve represents the spiking output of the cell. The output curve of 10-3, evaluated in this way, was very similar to the input curve from most stimulus orientations. However, there was a region in the lower left quadrant in which synaptic input seemed substantially larger than spiking output; i.e., the cell was depolarized to approximately the same level as in responses at other positions, but only a few spikes were produced. Therefore, the same apparent depolarization evoked at these stimulus orientations was much less effective in eliciting action potentials.

There could be several resons for this discrepancy. The first, and most obvious, is that the relative amplitudes of synaptic inputs, as observed in the cell body, will be different from their relative amplitudes summed at the SIZ. Recordings made at the soma are likely to overestimate the relative contribution of the proximal dendrite $Z$ to the overall response. Second, co-activation of inhibitory inputs that synapse onto dendrite $X$ would "shunt" the contribution of dendrite $Z$ 's inputs to spike initiation.

In order to test these possibilities, the input/output $(\mathrm{I} / \mathrm{O}) \mathrm{re}$ lationships for inputs to 10-3 were examined for each cercus independently. In Figure 5, $B$ and $C$, directional sensitivity plots based on spike counts and PSP areas are superimposed for each cercus. In both experiments, the directional sensitivity with both cerci intact was measured first; then 1 ccrcus was covered with Vaseline and the sensitivity was measured a second time. The curves were normalized to control responses from each experiment. Thus each curve represents the contribution of inputs to 10-3 from 1 cercus to the control response.

The $\mathrm{I} / \mathrm{O}$ relationship for inputs onto dendrite $\mathrm{X}$ revealed little if any discrepancy between the area under each response and the number of spikes produced $(n=4)$ (Fig. 10B). The directional sensitivity of the cell was nearly identical when measured with the 2 different methods. The $\mathrm{I} / \mathrm{O}$ relationship for inputs to dendrites $\mathrm{Y}$ and $\mathrm{Z}$ showed a large difference between synaptic inputs and the output produced $(n=5)$. The outer solid curve obtained by taking the area under each response is biased towards inputs from the rear and left side of the animal. The inner dashed curve, which represents spiking output, exhibits a reduced directional selectivity. By comparing the relative number of spikes produced by inputs from the left cercus (Fig. 10B) to the right cercus (Fig. 10C), it was obvious that inputs to dendrite $X$ via the left cercus were far more effective for spike initiation than inputs to dendrites $\mathrm{Y}$ and $\mathrm{Z}$ combined. This is easily understood, since dendrite $X$ is located significantly closer to the SIZ than either dendrite $\mathrm{Y}$ or $\mathrm{Z}$. As a result, more action potentials would be produced by input to dendrite $X$. Thus, dendrite $X$ appears to have a much larger influence on the directionally selective spiking output of this neuron than either of the other 2 dendrites. These experiments reveal that the directional sensitivity, unlike the receptive field of a neuron, is directly related to the location of the SIZ.

\section{Discussion}

This work demonstrates a strong correlation between the dendritic anatomy of interneuron 10-3 and its directionally selective response properties. The position of each dendrite with respect to the highly ordered primary afferent projection determines which excitatory inputs the cell can receive. By altering the neuron's structure in situ, we have shown that this structurefunction correlation is causally related to the excitatory receptive field of this interneuron. The experiments show that each dendritic branch of 10-3 receives input from a different class of afferents, and is therefore maximally sensitive to a different wind direction. These inputs in combination produce the overall excitatory receptive field of the neuron.

Two separate techniques were used to determine the receptive fields of individual dendrites: reducing afferent input to single classes of hairs, and selective laser photoinactivation. Data were compared for each dendrite (Fig. 11). When dendrite $\mathrm{Z}$ and the soma were isolated from the rest of the dendritic field (Jacobs and Miller, 1985), the directionality of excitatory input matched that of medial T hairs (Fig. $11 \mathrm{~A}$ ). The fact that their curves are so similar demonstrates that medial $\mathrm{T}$ hairs on the cercus ipsilateral to the soma provided the excitatory input to dendrite $Z$. This result is the most direct demonstration of the directional sensitivity of an individual dendrite in that the photoinactivation experiment isolates the dendrite from the rest of the cell.

The directional sensitivity of dendrite $Y$ can be characterized by a consideration of the photoinactivation experiment presented in Figure 5. In this experiment, the cercus contralateral to the soma was blocked, leaving the cercus ipsilateral to the soma intact. Thus, only dendrites $\mathrm{Y}$ and $\mathrm{Z}$ wcre receiving dircet afferent input. By subtracting the response after dendrite $Y$ was photoinactivated from the control curve, the response attributed to dendrite Y was obtained (Fig. 11C). This "difference" curve represents the contribution of dendrite $Y$ to the overall receptive field. This resembles the directionality when only anterior $\mathrm{L}$ hairs on the cercus ipsilateral to the soma were free to move (dashed line).

The directional sensitivity of dendrite $X$ was determined in 2 ways: (1) by measuring the response of the neuron with stimuli 


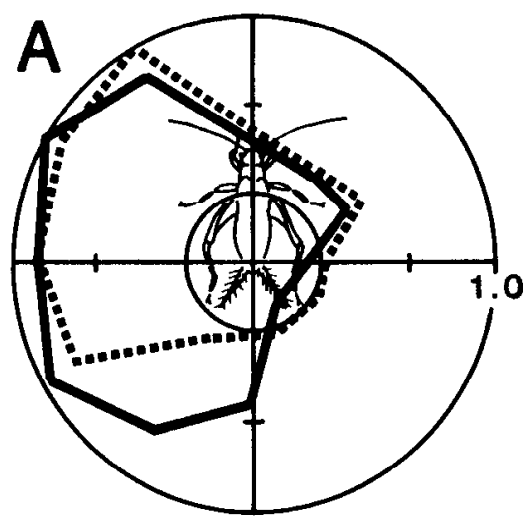

BOTH CERCI

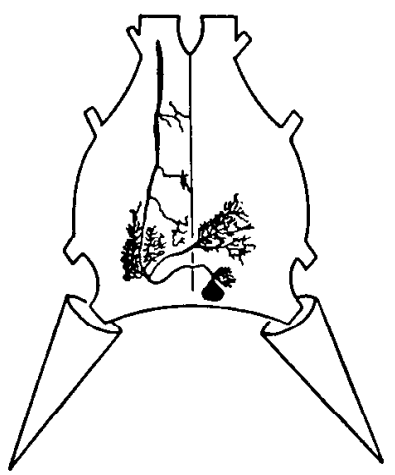

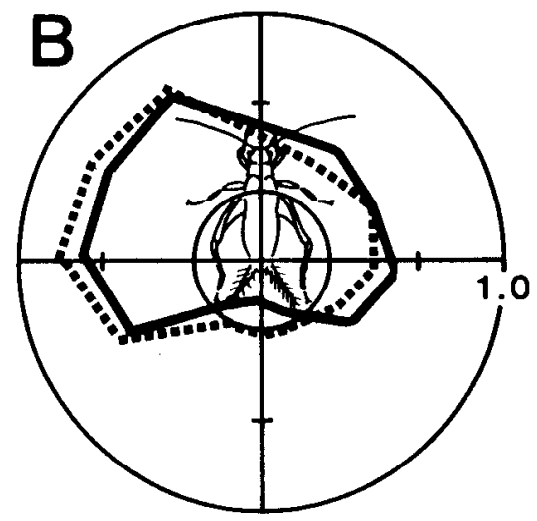

CERCUS CONTRALATERAL TO SOMA

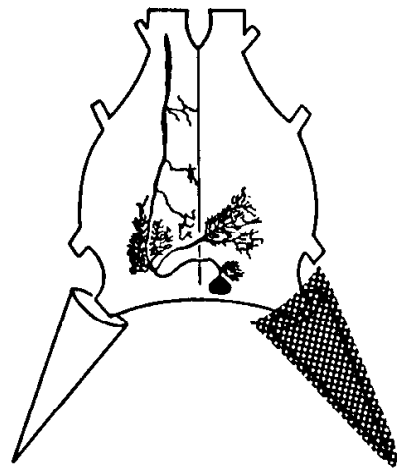

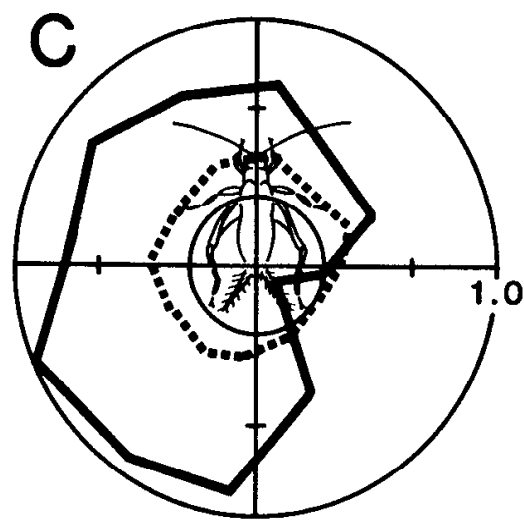

CERCUS IPSILATERAL TO SOMA

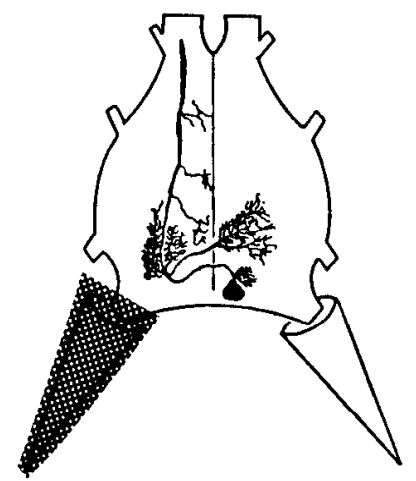

Figure 10. Input/output relationship of 10-3, derived by measuring both the area under each response and counting the number of action potentials for the first $100 \mathrm{msec}$ after stimulus onset. The solid lines represent area measurements normalized to control values; dashed lines represent the number of action potentials normalized to control values. $A$, I/O relationship of 10-3 for inputs to $10-3$ with both cerci intact, scaled to the maximum value. $B, \mathrm{I} / \mathrm{O}$ relationship for inputs to $10-3$ from the cercus contralateral to the soma. Both curves were normalized to a control with both cerci intact (not shown). $C, \mathrm{I} / \mathrm{O}$ relationship for inputs to 10-3 from the cercus ipsilateral to the soma. Both curves were normalized to a control with both cerci intact (not shown).

to a patch of lateral $\mathrm{T}$ afferents (Fig. $11 \mathrm{~B}$, dashed line), and (2) by recording the directional sensitivity with only the cercus contralateral to the soma intact (solid line). The range of wind stimulus orientations over which the neuron was most sensitive was nearly identical in the 2 experiments. Therefore, the main excitatory input to 10-3 from this cercus was mediated through lateral $T$ afferents that synapsed selectively with dendrite $X$.

Thus, by comparing 2 independent but complementary techniques, we can see that each dendrite of interneuron 10-3 has a distinct receptive field. The overall receptive field of this interneuron results, to a first-order approximation, from a summation of these excitatory inputs.

However, the findings presented in Figure 10 indicate that the cell's directional sensitivity cannot be precisely predicted from a direct linear summation of these excitatory inputs. Three factors could contribute to this nonlinearity of summation. First, if any membrane near the input synapses has regenerative properties, then doubling the amplitude of excitatory input might activate regenerative currents that would augment the synaptic currents. This would result in a positive nonlinearity, i.e., a response greater than double the initial response. Second, if the membrane were purely passive, activation of an excitatory synapse would bring the membrane potential closer to the synaptic equilibrium potential. Doubling the amplitude of the synaptic conductance change would then cause less than twice the voltage response, since the driving potential would be effectively reduced. Third, co-activation of an excitatory input with a strong inhibitory input (with a reversal potential near resting potential) would sum to an unpredictable, and apparently "nonlinear," intermediate value.

To understand how these factors might influence the directional sensitivity of 10-3, consider first the responses recorded in region $1-5$ in Figure 8 . These responses are thought to result from the summation of excitatory afferent inputs to all 3 dendrites. The linear sum of the responses resulting from stimuli to single cerci (dashed curve) results in a value that is larger than the control (solid curve). This is most likely due to a nonlinear summation of excitatory inputs near their excitatory reversal potential. The region of the curve at position 6 has been shown previously (Fig. 5) to activate inhibitory inputs to the interneuron. Here, the summed response was larger than the control. This is because the amplitude of the inhibitory response, as measured by the area under the voltage deflection, did not accurately reflect the strength of that inhibitory input. A large inhibitory conductance change might not cause a significant hyperpolarization, since the reversal potential may be near the resting potential. However, if it were co-activated with an excitatory conductance change, it could be a very effective shunt, causing an apparently nonlinear cancellation of the excitatory postsynaptic potential. Finally, at position 7 , an unexpected result was obtained. This region of the curve was thought to be due primarily to the contribution of excitatory inputs to dendrite Y. However, the summed response was larger than the control, indicating a co-activation of an inhibitory response masked by 

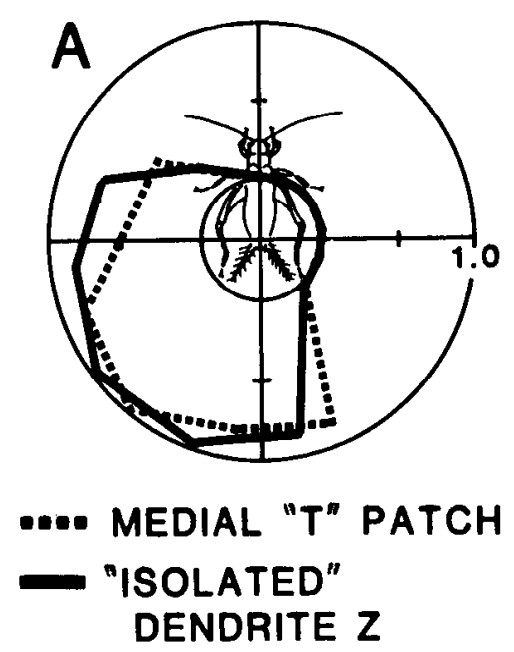
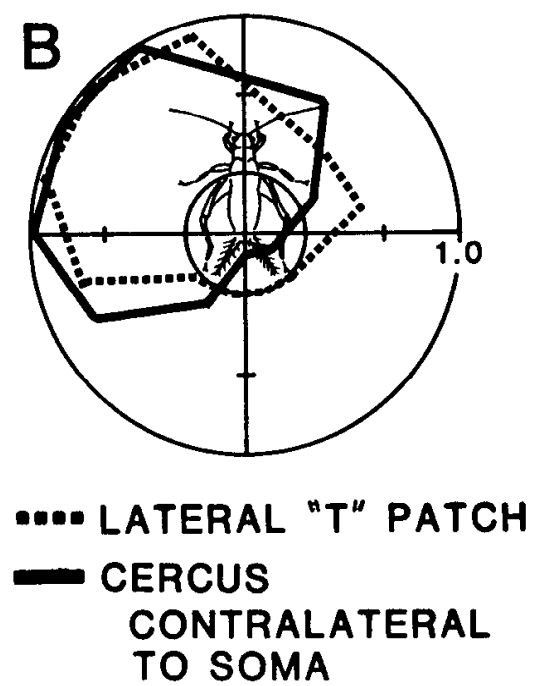
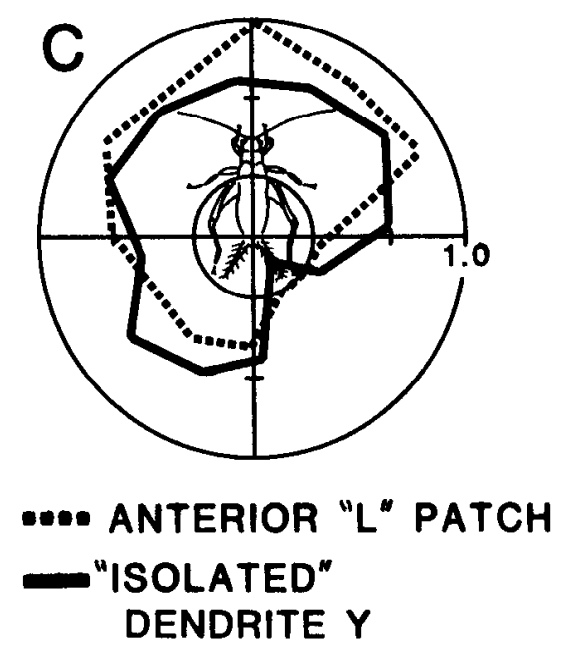

Figure 11. Comparison of directional sensitivities of 10-3's dendrites, obtained with different methods. $A$, Directional sensitivity of dendrite Z. Dotted line represents the response of 10-3 to a patch of medial "T" hairs (from Fig. 4C). Solid line represents the response of the cell when dendrite $\mathrm{Z}$ was isolated by photoinactivation (Fig. $5 D$, dotted line). $B$, Directional sensitivity of dendrite X. Dotted line represents response of $10-3$ to a patch of lateral "T" hairs (from Fig. $4 A$ ). Solid line rcpresents response to inputs from the intact cercus contralateral to the cell body. $C$, Directional sensitivity of dendrite Y. Dashed line represents response of 10-3 to a patch of anterior " $L$ " hairs on the cercus ipsilateral to the soma (from Fig. $4 B$ ). Solid line represents directional sensitivity of dendrite $Y$ determined by photoinactivation. This curve was obtained by subtracting the dashed line from the solid line in Figure 5.

excitation. This result was corroborated by the experiments, illustrated in Figure 7 , in which lateral $\mathrm{T}$ hairs on the cercus ipsilateral to the soma were shown to activate polysynaptic inhibitory inputs.

We have analyzed how 2 additional aspects of the interneuron's structure affect the integration of synaptic inputs. First, the relative efficacies of excitatory inputs to each dendrite are scaled according to the electrotonic distance between each input site and the SIZ. Second, inhibitory inputs act to sharpen the directional response of the cell by suppressing excitatory inputs outside the range of the cell's optimal directional response. The location of inhibitory inputs with respect to the individual dendrites and to the SIZ can effectively limit the spiking output of the cell and thus enhance directional sensitivity.

\section{Location of the spike-initiating zone}

The most probable location of the SIZ was shown to be at or very near the junction between the axon and the neurite segments linking all 3 dendritic arborizations. The proximity of an individual dendritic branch to the SIZ affects its relative contribution to the spiking output of the cell. Stimuli to dendrite $\mathrm{X}$ were found to be the most effective-a direct result of their proximity to the SIZ. The relative strengths of inputs to dendrites $Y$ and $Z$ were found to be less than those to $X$, though their absolute strengths could not be quantitatively determined.

A qualitative estimate of relative synaptic efficacies can be obtained by considering the patch experiments shown in Figure 4. Relative efficacies can be measured by the number of action potentials produced during a wind stimulus oriented in the optimal direction for each patch of hairs. If the response of 10-3 for each patch is compared (see Fig. 4), the dendrites can then be ranked in order of relative efficacy for spike initiation: dendrite $\mathrm{X}>$ dendrite $\mathrm{Y}>$ dendrite $\mathrm{Z}$. However, this is a rather crude measure, since "similar" amounts of synaptic current (i.e., 4 hairs in each patch) were injected into dendrites of widely different surface areas. Also, different patches might differ in synaptic efficacy; the number of synapses onto neuron 10-3 from each patch of hairs might be different. Despite these drawbacks, this finding suggests that synapses onto dendrite $X$ are the most effective for spike initiation. This corroborates the observation that inputs to dendrite $\mathrm{X}$ through the cercus contralateral to the soma (Fig. 10) are responsible for the majority of action potentials, and therefore crucial to the determination of the directionally selective output of this neuron.

\section{Source of inhibitory inputs onto 10-3}

The other major determinant of directional selectivity in this system is inhibitory input. The degree to which the excitatory response is suppressed appears to be a function of the balance between the number of afferents that provide excitation versus those that activate inhibition. For example, with both cerci intact, stimulus orientations optimal for dendrite $Z$ also activate the inhibitory pathway via afferents on the opposite cercus. In contrast, stimuli optimal for dendrite $\mathrm{X}$ do not co-activate either class of afferent that activates inhibition. Therefore, excitatory inputs to dendrite $X$ are not suppressed by inhibition, whereas inputs to dendrite $Z$ are suppressed. Consequently, the excitatory response of the interneuron is only suppressed within a specific range of stimulus orientations. Activation of posterior $\mathrm{L}$ hairs by wind from the rear of the animal or of lateral $\mathrm{T}$ hairs on the cercus ipsilateral to the soma was most effective in activating the inhibitory pathways.

The activation of inhibitory inputs to 10-3 studied by Levine and Murphey $(1980 \mathrm{a}, \mathrm{b})$ differs from that presented here, which can be attributed to the method used in their experiments to stimulate the afferents. The main differences between the 2 studies have to do with inputs to 10-3 from the cercus ipsilateral to the soma. Levine and Murphey (1980a, b) identified L hairs as providing excitatory input and $T$ hairs as mediating inhibition. This study, using wind stimuli, showed that $\mathrm{T}$ hairs provided excitation to dendrite $Z$, that anterior $L$ hairs provided excitation to dendrite $Y$, and that inhibition was activated via posterior $\mathrm{L}$ hairs on both cerci and lateral $\mathrm{T}$ hairs on the cercus ipsilateral to the soma (see Fig. 7). A model of the directional sensitivity of 10-3 put forth by Kamper (1985) is also in error with respect to the connectivity patterns both of excitatory and inhibitory inputs. 

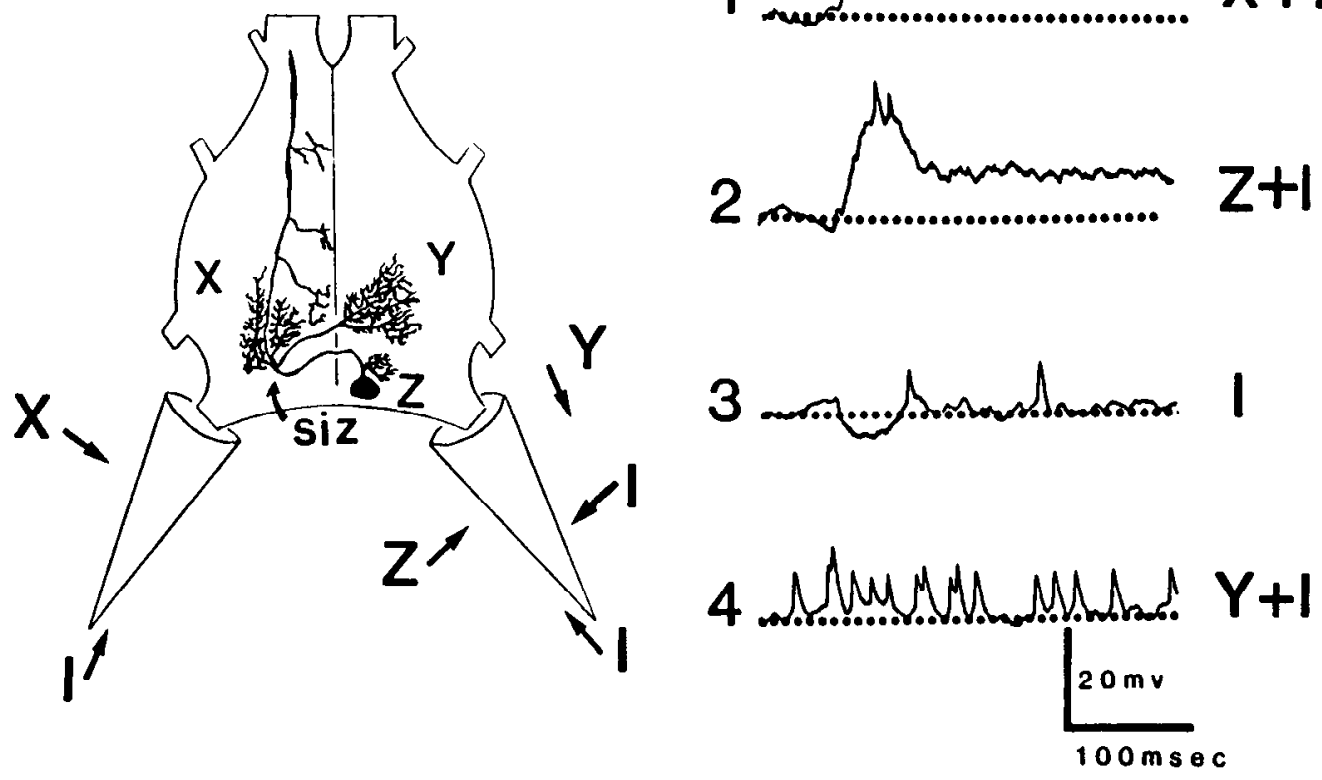

Figure 12. A cellular mechanism of directional sensitivity. Schematic diagram of 10-3 with all excitatory and inhibitory inputs indicated. Letters $(X$, $Y, Z)$ adjacent to sample records indicate excitatory inputs to each dendrite and co-activation of inhibitory inputs $(I)$.

\section{Location of inhibitory inputs on interneuron 10-3}

The location of the inhibitory synapses on the dendrites of 10-3 is of crucial importance to the integration of excitatory inputs. As first shown by Rall (1964), the most efficient location for inhibitory synapses would be at or near the SIZ of the neuron. In 10-3, inhibitory synapses to branch X near the SIZ could limit the spiking output of the cell very effectively. Since the excitatory synaptic current generated on any of the other dendrites must flow to this region, an inhibitory shunt could prevent the neuron from firing in response to stimuli from several orientations. Thus, relative suppression of excitatory inputs from each individual dendrite need not be achieved by activation of inhibitory synapses on that dendrite. In order to selectively suppress excitatory inputs from a particular dendrite, inhibitory inputs located near the SIZ must be co-activated at the same stimulus orientations that evoke excitatory afferent input to that dendrite. This co-activation is accomplished through a different class of afferents from those that provide the excitatory input. The experiments described here have demonstrated that (1) stimuli optimal for dendrite $\mathrm{X}$ do not co-activate inhibition, (2) stimuli optimal for dendrite $Y$ co-activate weak inhibition, and (3) stimuli optimal for dendrite $\mathrm{Z}$ co-activate strong inhibition. This selective suppression of excitatory inputs acts to "sharpen" the directional sensitivity of the neuron.

Although, to date, no inhibitory interneurons have been identified in this system, on the basis of what has been demonstrated in the experiments reported here, the structure of a putative wind-sensitive inhibitory interneuron can be predicted. This interneuron should have input branches located along the ventral midline in a position to receive excitation from posterior $L$ hairs, and an output region that would overlap with dendrite $\mathrm{X}$ of interneuron 10-3.

\section{A cellular mechanism of directional sensitivity}

An anatomical analysis of this neuron allows a prediction of its receptive field, yet gives no indication as to the relative weighting of each of the dendrites in terms of controlling the spiking output of the cell. In fact, it would appear from an analysis of Figure 10 that this cell could function almost "normally" (i.e., have the same general directional sensitivity) with half of its inputs blocked, as long as the inputs close to the SIZ were intact. We have shown that inhibition and electroanatomy are very important determinants of directional sensitivity in this cell. The structure/function analysis of 10-3 described in this paper incorporates these factors, and provides a precise framework from which to analyze the behavior of the neuron. If the analysis is correct, it should be possible to explain the response properties of 10-3 to a wind stimulus from any orientation.

To test our understanding of this system, 4 responses of 10-3 were chosen from an experiment in which both cerci were intact. Each response shown in Figure 12 was labeled according to the activation of excitatory input to each dendrite $(X, Y, Z)$, and activation of inhibitory inputs (I) according to wind stimulus orientation. Record 1 demonstrates the response of 10-3 to activation of excitatory inputs to all 3 dendrites simultaneously. This stimulus orientation provided the maximum synaptic current to the SIZ, the majority of which was from inputs to dendrite $\mathrm{X}$. Consequently, the recorded depolarization was maximal and more spikes were produced than for any other stimulus orientation. Record 2 demonstrates co-activation of excitatory inputs to dendrite $Z$ (on the cercus ipsilateral to the soma) and polysynaptic inhibitory inputs. The depolarization was about $75 \%$ of the amplitude of that in record 1 ; however, only 2 spikes were produced. Since the excitatory input was coming in very close to the recording site, there was little decrement of the synaptic potential. However, simultaneous activation of inhibitory synapses near the SIZ could shunt all but 2 of the spikes that would otherwise have been produced by the depolarization. Record 3 demonstrates the activation of the inhibitory pathway. The stimulus orientation was optimal for activation of inhibition by inputs from both cerci. Record 4 demonstrates the activation of excitatory inputs to dendrite $Y$. Synaptic potentials to this dendrite may have been too far away, electrotonically, to be recorded in the cell body, so the response was limited to passively propagated spikes initiated at the SIZ. The spike frequency was half that observed in record 1 and could have been due to less total synaptic current, which is a function of coactivation of weak inhibitory inputs and passive attenuation en route to the SIZ. 
Comparisons with sensory systems in other animals

The functional implications of dendritic geometry have been studied in a variety of sensory systems subserving several different sensory modalities. In systems where primary afferents are arranged topographically, the receptive fields of sensory interneurons are highly correlated with the position and shape of their dendritic fields. For example, hair follicle afferents from the cat hind limb terminate topographically in the dorsal horn of the spinal cord according to the location of their peripheral fields (Brown et al., 1977). The dendritic trees of spinocervical tract interneurons occupy discrete regions within the afferent array and their peripheral receptive fields are correlated with the location of the dendritic arbor (Brown et al., 1980a, b).

In the cat visual system, dendritic geometry is thought to influence the receptive field characteristics of cells at several levels in the visual pathway. In the lateral geniculate nucleus, $X$ cells have dendrites oriented perpendicular to the laminae, which is thought to maximize the preservation of retinotopy and thus enhance the cells' responsiveness to high spatial frequencies (Stanford et al., 1983). W cell dendrites are oriented parallel to the laminae, which ensures convergent input from many afferents and results in a large representation of visual space that may be important in ocular dominance mechanisms (Stanford et al., 1983). In area 17, first-order neurons have dendritic fields that are elongated parallel to the pial surface. Since the sensory input is organized topographically, it is suggested that dendritic field shape could determine the receptive field shape of these cells (Tieman and Hirsch, 1985).

Inputs to acoustic interneurons in the nucleus laminaris of the chick auditory system are organized tonotopically across the nucleus, and the position of an interneuron is correlated with its optimal response frequency (Rubel and Parks, 1975; Young and Rubel, 1983). The dendrites of these cells are spatially segregated into dorsal and ventral regions, which divide inputs of the same frequency from the ipsilateral and contralateral ears, respectively. In this system, the spatial segregation of inputs is thought to be involved in binaural processing of sound, and thus dendritic position plays more of an integrative role in signalprocessing rather than specifying the optimal frequency of the cell.

It is important to distinguish between one role of neuronal structure-determining the overall receptive field of a neuronand another-optimizing the sensitivity of the cell within its receptive field. Optimal sensitivity has been demonstrated in many systems to be a function of the effects of inhibitory inputs and the integrative properties of the cell. For example, the orientation selectivity of neurons in the cat visual system has been shown to be enhanced in both adult cats (Sillito, 1975; Sillito et al., 1980) and young kittens (Sato and Tusumoto, 1984) by GABAergic inhibition. In the crayfish mechanosensory system, inhibitory inputs from nonspiking interneurons play a major role in shaping the directional responses of these neurons (Reichart et al., 1983). The function of the inhibitory interneurons is to suppress the response of the directionally selective projection interneurons to stimuli common to both sides of the animal, and to accentuate the difference between stimuli to either side.

The results presented here on interneuron 10-3 have shown that a major determinant of the directional sensitivity of the cell is the location of the spike-initiating zone with respect to the distribution of excitatory and inhibitory inputs. Thus the cell responds maximally to inputs within a small region of its overall receptive field. A quantitative study of crayfish visual interneurons has yielded a similar result (Kirk et al., 1983): the receptive field of these cells is correlated with the position of the dendritic field, yet maximal sensitivity occurs within a small sector of the field and is directly correlated with dendritic density.
To conclude: It is likely that the mechanisms underlying directional sensitivity and sensory processing that are described here in detail for cricket interneurons operate in a variety of vertebrate and invertebrate systems. It is now apparent that there are several levels at which a neuron's function must be related to its structure. In this system, the position of dendrites with respect to the topography of the afferent map determines the types of excitatory input the cell can receive. The location of the SIZ determines the relative weighting of each dendrite and inhibition acts to selectively suppress particular excitatory inputs. All 3 mechanisms work in combination to produce a directionally selective output unique to that interneuron.

\section{References}

Bacon, J. P., and J. S. Altman (1977) A silver intensification method for cobalt filled neurones in wholemount preparations. Brain Res. 138: 359-363.

Bacon, J. P., and R. K. Murphey (1984) Receptive fields of cricket (Acheta domesticus) interneurones are related to their dendritic structure. J. Physiol. (Lond.) 352: 601-623.

Brown, A. G., P. K. Rose, and P. J. Snow (1977) The morphology of hair follicle afferent fibre collaterals in the spinal cord of the cat. $J$. Physiol. (Lond.) 272: 779-797.

Brown, A. G., R. E. Fyffe, R. Noble, P. K. Rose, and P. J. Snow (1980a) The density, distribution and topographical organization of the spinocervical tract neurones in the cat. J. Physiol. (Lond.) 300: 409428

Brown, A. G., P. K. Rose, and P. J. Snow (1980b) Dendritic trees and cutaneous receptive fields of adjacent spinocervical tract neurones in the cat. J. Physiol. (Lond.) 300: 429-440.

Callec, J. J., J. C. Guillet, Y. Pinchon, and J. Boistel (1971) Further studies on synaptic transmission in insects. II. Relations between sensory information and its synaptic integration at the level of a single giant axon in the cockroach. J. Exp. Biol. 55: 123-149.

Edwards, J. S., and J. Palka (1974) The cerci and abdominal giant fibers of the house cricket Acheta domesticus. I. Anatomy and physiology of normal adults. Proc. R. Soc. Lond. [Biol.] 185: 83-103.

Gnatzy, W., and J. Tautz (1980) Ultrastructurc and mechanical properties of an insect mechanoreceptor: Stimulus transmitting structures and sensory apparatus of the cercal filiform hairs of Gryllus. Cell Tissue Res. 213: 441-463.

Jacobs, G. A., and J. P. Miller (1985) Functional properties of individual neuronal branches isolated in situ by laser photoinactivation. Science 228: 344-346.

Kamper, G. (1985) Processing of species specific low frequency song components by interneurons in crickets. In Acoustic and Vibrational Communication in Insects, H. Kalmring and K. Elsner, eds., pp. 4754, Parey, Berlin.

Kater, S. B., C. S. Cohan, G. A. Jacobs, and J. P. Miller (1985) Image intensification of stained, functioning and growing neurons. In Optical Methods in Cell Physiology, P. DeWeer and B. Salzburg, eds., pp. 3150, Wiley, New York.

Kirk, M. D., B. Waldrop, and R. M. Glantz (1983) A quantitative correlation of contour sensitivity with dendritic density in an identified visual neuron. Brain Res. 274: 231-237.

Levine, R. B., and R. K. Murphey (1980a) Loss of inhibitory synaptic input to cricket sensory interneurons as a consequence of partial deafferentation. J. Neurophysiol. 43: 383-394.

Levine, R. B., and R. K. Murphey (1980b) Pre- and postsynaptic inhibition of identified giant interneurons in the cricket (Acheta domesticus). J. Comp. Physiol. 135: 269-282.

Matsumoto, S. G., and R. K. Murphey (1977) The cercus-to-giant interneuron system of crickets. IV. Patterns of connectivity between receptors and the medial giant interneuron. J. Comp. Physiol. 119: 319-330.

Millcr, J. P., and G. A. Jacobs (1984) Relationships between neuronal structure and function. J. Exp. Biol. 112: 129-145.

Miller, J. P., and A. I. Selverston (1979) Rapid killing of single neurons by irradiation of intracellularly injected dye. Science $185: 181-183$.

Murphey, R. K., A. Jacklet, and L. Schuster (1980) A topographic map of sensory cell terminal arborizations in the cricket CNS: Correlation with birthday and position in a sensory array. J. Comp. Neurol. 191: 53-64. 
Murphey, R. K., and C. A. Lemere (1984) Competition controls the growth of an identified axonal arborization. Science 224: 1352-1355.

O'Shea, M., and M. E. Adams (1981) Pentapeptide (proctolin) associated with an identified neuron. Science 4507: 567-569.

Palka, J., R. Levine, and M. Schubiger (1977) The cercus-to-giant interneuron system of crickets. I. Some attributes of the sensory cells. J. Comp. Physiol. 119: 267-283.

Rall, W. (1964) Theoretical significance of dendritic trees for neuronal input-output relations. In Neural Theory and Modelling, R. F. Reiss, ed., pp. 73-79, Stanford University Press, Stanford, CA.

Reichart, H., M. R. Plummer, and J. J. Wine (1983) Identified nonspiking local interneurons mediate non-recurrent, lateral inhibition of crayfish mechanosensory interneurons. J. Comp. Physiol. 151: 261276.

Rubel, E. W., and T. N. Parks (1975) Organization and development of brain stem auditory nuclei of the chicken: Tonotopic organization of $\mathrm{n}$. magnocellularis and $\mathrm{n}$. laminaris. J. Comp. Neurol. 164: 411434.

Sato, H., and T. Tsumoto (1984) GABAergic inhibition already operates on a group of neurones in the kitten visual cortex at the time of eye opening. Dev. Brain Res. 12: 311-315.

Shepherd, D., and R. K. Murphey (in press) Quantal analysis of competition in the CNS of crickets. J. Neurosci.

Sillito, A. M. (1975) The contribution of inhibitory mechanisms to the receptive field properties of neurones in the striate cortex of the cat. J. Physiol. (Lond.) 250: 305-329.

Sillito, A. M., J. A. Kemp, I. A. Milson, and N. Bernardi (1980) A reevaluation of the mechanisms underlying simple cell orientation selectivity. Brain Res. 194: 517-520.

Stanford, L. R., M. J. Friedlander, and S. M. Sherman (1983) Morphological and physiological properties of geniculate $\mathrm{W}$ cells of the cat: A comparison with X and Y cells. J. Neurophysiol. 50: 582-608.

Tieman, S. B., and H. V. B. Hirsch (1985) Role of dendritic fields in orientation selectivity. In Models of Visual Cortex, D. Rose and V. G. Dobson, eds., pp. 432-442, Wiley, New York.

Tieman, D. G., R. K. Murphey, J. T. Schmidt, and S. B. Tieman (in press) A computer assisted video technique for preparing high resolution pictures and stereograms from thick specimens. Brain Res.

Tobias, M., and R. K. Murphey (1979) The response of cercal receptors and identified interneurons in the cricket (Acheta domesticus) to air streams. J. Comp. Physiol. 129: 51-59.

Walthall, W. W., and R. K. Murphey (1986) Positional information, compartments and the cercal system of crickets. Dev. Biol. 113:182200.

Young, S. R., and E. W. Rubel (1983) Frequency-specific projections of individual ncurons in chick brainstem auditory nuclei. J. Neurosci. 3: 1373-1378. 